\title{
Ecologia das comunidades de metazoários parasitos, do xaréu, Caranx hippos (Linnaeus) e do xerelete, Caranx latus Agassiz (Osteichthyes, Carangidae) do litoral do estado do Rio de Janeiro, Brasil ${ }^{1}$
}

\author{
José Luis Luque ${ }^{2}$ \\ Dimitri Ramos Alves ${ }^{2}$
}

\begin{abstract}
Community ecology of metazoan parasites of the crevalle jack, Caranx hippos (Linnaeus) and the horse-eye jack, Caranx latus Agassiz (Osteichthyes, Carangidae) from the coastal zone of the State of Rio de Janeiro, Brazil. Sixty specimens of Caranx hippos (Linnaeus, 1766) and fifty-five specimens of Caranx latus Agassiz, 1831 collected from the coastal zone of the State of Rio de Janeiro $\left(21-23^{\circ} \mathrm{S}, 41-45^{\circ} \mathrm{W}\right)$, Brazil, from October 1998 to October 1999, were necropsied to study their metazoan parasites. All specimens of $C$. hippos were parasitized, and the majority of specimens of $C$. latus $(96.4 \%)$ were parasitized by one or more metazoan species. Nineteen species of parasites were collected in $C$. hippos: 5 digeneans, 5 monogeneans, 2 cestodes, 5 nematodes, and 2 copepods. Seventeen species of parasites were collected in C. latus: 6 digeneans, 2 monogeneans, 3 cestodes, 4 nematodes, and 2 copepods. The endoparasites (digeneans, cestodes, and nematodes) were the majority, $76.4 \%$ and $63.3 \%$, of the total number of parasite specimens collected in C. hippos and C. latus, respectively. The monogeneans Allopyragraphorus hippos (Hargis, 1956) and Cemocotyle carangis (MacCallum, 1913) were the most dominant species with the highest parasitic prevalence in the parasite community of C. hippos and C. latus, respectively. The metazoan parasites of the two host species showed the typical overdispersed pattern of distribution. Bucephalus varicus Manter, 1940, A. hippos, Protomicrocotyle mirabilis (MacCallum, 1918), Cucullanus pulcherrimus Barreto, 1918, and Lernanthropus giganteus Krфyer, 1863 had a positive correlation only between the host's total length and abundance and/or prevalence in C. hippos. Bucephalus varicus, Tergestia pectinata (Linton, 1905), C. carangis, and Pseudoterranova sp. had a positive correlation between the host's total length and abundance and/or prevalence in C. latus. In C. hippos, the copepod Caligus robustus Bassett-Smith, 1898 had the highest values of prevalence and abundance in the female hosts. No parasite species showed influence of the host's sex on their prevalence and abundance in $C$. latus. The mean diversity and the parasite species richness of the parasite infracommunities of $C$. hippos and $C$. latus were not significantly different. Only the parasite species diversity of $C$. hippos was correlated with the host's total length; in both host species the parasite diversity did not showed differences in relation to the sex of the host. Only one pair of ectoparasite species, A. hippos - P. mirabilis, showed significant positive co-occurrence and covariation in the parasite infracommunities of $C$. hippos. Two endoparasite species, $B$. varicus - Parahemiurus merus (Linton, 1910), showed negative co-occurrence and positive covariation; and the pair B. varicus - Pseudoterranova sp. had positive co-occurrence and covariation in the
\end{abstract}

1) Trabalho desenvolvido com auxílios do CNPq, CAPES e FAPERJ.

2) Departamento de Parasitologia Animal, Curso de Pós-Graduação em Parasitologia Veterinária, Universidade Federal Rural do Rio de Janeiro. Caixa Postal 74508, 23851-970 Seropédica, Rio de Janeiro, Brasil. E-mail: jlluque@ufrrj.br 
infracommunities of $C$. latus. The values of qualitative and quantitative similarity coefficients between the parasite communities of $C$. hippos and $C$. latus were 55.5 and 30.4 , respectively. The parasite communities of $C$. hippos and C. latus were defined as closest to isolationist type because there are few evidences of interspecific associations or covariations. Additional parasitological studies on other species of carangid fishes from the South American Atlantic Ocean are needed to evaluate the structure of carangid parasite communities in the Neotropical region.

KEY WORDS. Carangidae, Caranx hippos, Caranx latus, parasitic ecology, community structure, marine fish, Brazil

Os peixes marinhos de Carangidae são espécies pelágicas de águas tropicais de superfície, que formam cardumes junto à costa; são carnívoros e alimentam-se basicamente de peixes, crustáceos e em menor escala de invertebrados planctônicos. Os carangídeos têm importância econômica e grande demanda nos mercados e peixarias do Rio de Janeiro (MENEZES \& FigueIREDo 1980). O xaréu Caranx hippos (Linnaeus, 1766) e o xerelete Caranx latus Agassiz, 1831, são espécies bastante freqüentes no litoral do estado do Rio de Janeiro e apresentam amplitudes de distribuição geográfica conhecidas superpostas, abrangendo o litoral do Oceano Atlântico na América do Norte e na América do Sul (MENEZES \& FigueIrEdo 1980; CarvalHo-Filho 1999).

Os hábitos alimentares destes peixes revelam sua grande potencialidade para participar como hospedeiros intermediários, ou definitivos em sistemas parasitários transmitidos troficamente. Isto reflete-se na literatura disponível sobre os parasitos de carangídeos no Oceano Atlântico da América do Norte, e já foi observado no Brasil para três espécies de guaiviras (Oligoplites spp.) no litoral do estado do Rio de Janeiro por TAKEMOTO et al. (1996).

Registros de parasitos do xaréu no Brasil são escassos e foram feitos por TRAVASSOS et al. (1966) e AMATO (1982, 1983). Alguns parasitos foram registrados no xerelete no litoral brasileiro por FERNANDES et al. (1985) e KOHN et al. (1992). Recentemente, LUQUE et al. (2000) registraram 24 espécies de metazoários parasitos de C. hippos e C. latus no Rio de Janeiro e fizeram uma revisão dos registros parasitários conhecidos para estas espécies na Região Neotropical. O único trabalho de características ecológicas foi feito por BASHIRULLAH \& RODRIGUEZ (1992) que estudaram a distribuição dos monogenéticos nas brânquias de $C$. hippos na Venezuela.

Os parasitos de peixes, constituem um excelente modelo para estudos sobre ecologia de comunidades. A possibilidade de obter numerosas réplicas e de quantificar a totalidade dos parasitos que estão distribuídos em diversos hábitats (locais de infecção) facilitam a detecção de padrões da dinâmica populacional e de relacionamentos interespecíficos (ROHDE 1991). Entretanto, recentes trabalhos sobre as comunidades parasitárias de peixes marinhos neotropicais indicam a presença de complexos de espécies insaturados e pouco ordenados, mostrando padrões heterogêneos quanto à sua estrutura, revelando a necessidade de continuar acumulando evidências da presença ou ausência de padrões de organização e de processos interativos (RoHde et al. 1995; CezAR \& Luque 1999; Luque \& ChAves 1999). 
Neste trabalho é apresentada uma análise das comunidades parasitárias de C. hippos e de C. latus, com o intuito de avaliar a dinâmica das suas infrapopulações parasitárias, examinando seus relacionamentos com alguns fatores bióticos como o comprimento total e o sexo dos hospedeiros, seus relacionamentos interespecíficos, similaridade e a diversidade das respectivas infracomunidades parasitárias.

\section{MATERIAL E MÉTODOS}

Entre outubro de 1998 e outubro de 1999, foram necropsiados 60 espécimes de C. hippos e 55 espécimes de C. latus, provenientes do litoral do estado do Rio de Janeiro $\left(21-23^{\circ} \mathrm{S}, 41-45^{\circ} \mathrm{W}\right)$. Os espécimes de C. hippos e de C. latus mediram $43,9 \pm 11,2(27-64)$ e $33,2 \pm 5,3(26-43) \mathrm{cm}$ de comprimento total, respectivamente. A determinação dos peixes foi feita de acordo com MENEZES \& FIGUEIREDo (1980).

A dominância de cada componente das infracomunidades parasitárias foi determinada mediante o cálculo da freqüência de dominância e da dominância relativa (número de espécimes de uma espécie/número total de espécimes de todas as espécies de cada infracomunidade), seguindo a metodologia de ROHDE et al. (1995). O quociente entre a variância e a abundância parasitária média (índice de dispersão) foi calculado para cada espécie de parasito com o intuito de determinar seu padrão de distribuição. O grau de agregação foi obtido através do cálculo do índice de Green (LUDWIG \& REYNOLDS 1988).

O coeficiente de correlação por postos de Spearman $r s$ foi usado para determinar possíveis correlações entre o comprimento total do hospedeiro e a abundância de infecção. O coeficiente de correlação de Pearson $r$ foi usado para determinar a possível correlação entre o comprimento total do hospedeiro e a prevalência parasitária, com prévia transformação angular dos dados de prevalência (ZAR 1996); no caso de C. hippos as amostras dos hospedeiros foram separadas em quatro intervalos de classe de $10 \mathrm{~cm}$, para C. latus foram separadas em cinco intervalos de classe de $4 \mathrm{~cm}$. A influência do sexo sobre a abundância e prevalência das infecções parasitárias foi testada pela aproximação normal $Z$ do teste $U$ de Mann-Whitney e pelo teste exato de Fisher $(F)$, respectivamente.

A diversidade parasitária de cada infracomunidade foi calculada através do índice de Brillouin $(H)$, sendo determinada sua possível variação em relação ao sexo (aproximação normal $Z$ do teste $U$ de Mann-Whitney) e ao comprimento total do hospedeiro (coeficiente de correlação por postos de Spearman $r s$ ). Foi calculado também para cada infracomunidade o índice de uniformidade baseado no índice de Brillouin $(J)$ (ZAR 1996). As possíveis associações interespecíficas entre pares de espécies co-ocorrentes foram determinadas através do qui-quadrado. Possível covariação entre a abundância parasitária das espécies que formavam as associações foram analisadas com o coeficiente de correlação por postos de Spearman $r s$ (LUDWIG \& REYNOLDS 1988). Possíveis diferenças entre a diversidade parasitária, riqueza parasitária e o número total de parasitos das comunidades parasitárias do xaréu e do xerelete foram testadas pela aproximação normal $Z$ do teste $U$ de Mann-Whitney (ZAR 1996).

A similaridade qualitativa e quantitativa entre as comunidades parasitárias de $C$. hippos e C. latus foi determinada pelo uso do coeficiente de similaridade de 
Sorenson (NERAASEN \& HOLMES 1975) e pelo coeficiente percentual de similaridade (Holmes \& PODESTA 1968), respectivamente.

A terminologia ecológica usada é a recomendada por BuSH et al. (1997). A análise incluiu somente as espécies com prevalência maior que 10\% (BusH et al. 1990). Todos os valores que correspondem à média de alguma variável são acompanhados do respectivo desvio padrão. O nível de significância estatística adotado foi $P \leq 0,05$.

\section{RESULTADOS}

\section{Componentes das comunidades parasitárias}

Foram determinadas 19 espécies de metazoários parasitos de $C$. hippos: 5 digenéticos, 5 monogenéticos, 2 cestóides, 5 nematóides e 2 copépodes. O monogenético Allopyragraphorus hippos (Hargis, 1956) foi a espécie mais prevalente (Tab. I) e apresentou a maior freqüência de dominância e o maior valor de dominância relativa média (Tab. III). Em C. latus, foram determinadas 17 espécies de metazoários parasitos: 6 digenéticos, 2 monogenéticos, 3 cestóides, 4 nematóides e 2 copépodes. O monogenético Cemocotyle carangis (MacCallum, 1913) foi a espécie mais prevalente e abundante (Tab. II) e apresentou a maior freqüência de dominância e o maior valor de dominância relativa média (Tab. IV).

Tabela I. Prevalência, amplitude da intensidade, intensidade média e abundância média dos metazoários parasitos de Caranx hippos do litoral do estado do Rio de Janeiro, Brasil.

\begin{tabular}{|c|c|c|c|c|}
\hline Parasito & $\begin{array}{l}\text { Prevalência } \\
\qquad \%)\end{array}$ & $\begin{array}{l}\text { Amplitude da } \\
\text { intensidade }\end{array}$ & $\begin{array}{l}\text { Intensidade } \\
\text { média }\end{array}$ & $\begin{array}{l}\text { Abundância } \\
\text { média }\end{array}$ \\
\hline \multicolumn{5}{|l|}{ Digenea } \\
\hline Bucephalus varicus Manter, 1940 & 38,4 & $1-90$ & 9,2 & 3,50 \\
\hline Lecithochirium microstomum Chandler, 1935 & 5,0 & $1-3$ & 2,0 & 0,10 \\
\hline Manteria brachyderus (Manter, 1940) & 20,0 & $1-33$ & 5,1 & 1,02 \\
\hline Stephanostomum ghanensis Fischthal \& Thomas, 1968 & 1,7 & $1-7$ & 7,0 & 0,10 \\
\hline \multicolumn{5}{|l|}{ Monogenea } \\
\hline Allopyragraphorus hippos (Hargis, 1956) & 51,6 & $1-17$ & 6,8 & 3,50 \\
\hline Allopyragraphorus sp. & 3,3 & - & 1,0 & 0,03 \\
\hline Cemocotyllela elongata (Meserve, 1938) & 18,3 & $1-8$ & 3,3 & 0,60 \\
\hline Cemocotyle noveboracensis Price, 1962 & 35,0 & $1-35$ & 5,8 & 2,03 \\
\hline Protomicrocotyle mirabilis (MacCallum, 1918) & 31,7 & $1-7$ & 2,7 & 0,90 \\
\hline \multicolumn{5}{|l|}{ Cestoidea } \\
\hline Nybelinia sp. (larva plerocercóide) & 6,7 & - & 1,0 & 0,10 \\
\hline Callitetrarhynchus gracilis (Rudolphi, 1819) (larva plerocercóide) & 1,6 & - & 1,0 & 0,01 \\
\hline \multicolumn{5}{|l|}{ Nematoda } \\
\hline Contracaecum sp. (larva) & 3,3 & $1-4$ & 2,5 & 0,04 \\
\hline Cucullanus pulcherrimus Barreto, 1918 & 11,7 & $1-4$ & 3,0 & 0,30 \\
\hline Philometra sp. & 8,3 & $1-605$ & 131,2 & 10,90 \\
\hline Pseudoterranova sp. (larva) & 6,7 & $1-2$ & 1,5 & 0,10 \\
\hline Raphidascaris sp. (larva) & 5,0 & $1-39$ & 27,3 & 1,30 \\
\hline \multicolumn{5}{|l|}{ Copepoda } \\
\hline Caligus robustus Bassett-Smith, 1898 & 16,7 & $1-3$ & 1,8 & 0,30 \\
\hline Lernanthropus giganteus Krфyer, 1863 & 10,0 & $1-4$ & 2,5 & 0,20 \\
\hline
\end{tabular}

Os endoparasitos (digenéticos, cestóides e nematóides) constituíram 76,4\% e $63,3 \%$ do total de parasitos coletados no xaréu e no xerelete respectivamente. Os componentes da comunidade parasitária de C. hippos e C. latus apresentaram o típico padrão de distribuição superdispersa (Tabs V, VI). 
Tabela II. Prevalência, amplitude da intensidade, intensidade média e abundância média dos metazoários parasitos de Caranx latus do litoral do estado do Rio de Janeiro, Brasil.

\begin{tabular}{|c|c|c|c|c|}
\hline Parasito & $\begin{array}{c}\text { Prevalência } \\
(\%)\end{array}$ & $\begin{array}{l}\text { Amplitude da } \\
\text { intensidade }\end{array}$ & $\begin{array}{l}\text { Intensidade } \\
\text { média }\end{array}$ & $\begin{array}{l}\text { Abundância } \\
\text { média }\end{array}$ \\
\hline \multicolumn{5}{|l|}{ Digenea } \\
\hline Bucephalus varicus Manter, 1940 & 32,7 & $1-34$ & 9,0 & 2,9 \\
\hline Lecithochinum microstomum Chandler, 1935 & 7,2 & $1-4$ & 1,7 & 0,1 \\
\hline Parahemiurus merus (Linton, 1910) & 52,7 & $1-18$ & 5,5 & 2,9 \\
\hline Stephanostomum ghanensis Fischthal \& Thomas, 1968 & 1,8 & - & 1,0 & 0,1 \\
\hline Tergestia pectinata (Linton, 1905) & 12,7 & $1-13$ & 6,1 & 0,7 \\
\hline \multicolumn{5}{|l|}{ Monogenea } \\
\hline Cemocotyle carangis (MacCallum, 1913) & 60,0 & $1-49$ & 14,6 & 8,7 \\
\hline Pseudomazocraes selene Hargis, 1957 & 34,5 & $1-8$ & 3,2 & 1,1 \\
\hline \multicolumn{5}{|l|}{ Cestoidea } \\
\hline Callitetrarhynchus gracilis (Rudolphi, 1819) (larva plerocercóide) & 5,4 & $1-15$ & 6,3 & 0,3 \\
\hline Nybelinia sp. (larva plerocercóide) & 20,0 & $1-13$ & 4,8 & 0,9 \\
\hline Scolex pleuronectis Müller, 1788 (metacestóide) & 3,6 & - & 1,0 & 0,1 \\
\hline \multicolumn{5}{|l|}{ Nematoda } \\
\hline Anisakis sp. (larva) & 1,8 & - & 1,0 & 0,1 \\
\hline Contracaecum sp. (larva) & 18,2 & $1-20$ & 6,2 & 1,2 \\
\hline Hysterothylacium sp. (larva) & 3,6 & $1-3$ & 2,5 & 0,1 \\
\hline Pseudoterranova sp. (larva) & 32,7 & $1-77$ & 22,3 & 7,3 \\
\hline \multicolumn{5}{|l|}{ Copepoda } \\
\hline Caligus robustus Bassett-Smith, 1898 & 1,8 & - & 1,0 & 0,1 \\
\hline Lernanthropus giganteus Krфyer, 1863 & 1,8 & - & 1,0 & 0,1 \\
\hline
\end{tabular}

Tabela III. Freqüência de dominância e dominância relativa média dos componentes das infracomunidades de metazoários parasitos de Caranx hippos do litoral do estado do Rio de Janeiro, Brasil.

\begin{tabular}{lccc}
\hline \multicolumn{1}{c}{ Parasito } & Freqüência de dominância & $\begin{array}{c}\text { Freqüència de dominância } \\
\text { compartilhada }\end{array}$ & Dominância relativa mèdia \\
\hline Allopyragraphorus hippos & 19 & 1 & $0,252 \pm 0,303$ \\
Cemocotyllela elongata & 1 & 0 & $0,038 \pm 0,132$ \\
Cemocotyle noveboracensis & 8 & 0 & $0,142 \pm 0,306$ \\
Protomicrocotyle mirabilis & 1 & 0 & $0,057 \pm 0,104$ \\
Bucephalus varicus & 13 & 0 & $0,182 \pm 0,312$ \\
Manteria brachyderus & 3 & 1 & $0,202 \pm 1,166$ \\
Parahemiurus merus & 8 & 0 & $0,113 \pm 0,234$ \\
Cucullanus pulcherrimus & 0 & 0 & $0,009 \pm 0,047$ \\
Caligus robustus & 1 & 0 & $0,043 \pm 0,148$ \\
Lernanthropus giganteus & 0 & 0 & $0,004 \pm 0,021$ \\
\hline
\end{tabular}

Tabela IV. Freqüência de dominância e dominância relativa média dos componentes das infracomunidades de metazoários parasitos de Caranx latus do litoral do estado do Rio de Janeiro, Brasil.

\begin{tabular}{lccc}
\hline \multicolumn{1}{c}{ Parasito } & Frequència de dominância & $\begin{array}{c}\text { Frequência de dominância } \\
\text { compartilhada }\end{array}$ & $\begin{array}{c}\text { Dominância relativa média } \\
\text { Bucephalus varicus }\end{array}$ \\
Parahemiurus merus & 8 & 0 & $0,162 \pm 0,304$ \\
Tergestia pectinata & 7 & 0 & $0,179 \pm 0,286$ \\
Cemocotyle carangis & 3 & 0 & $0,048 \pm 0,158$ \\
Pseudomazocraes selene & 21 & 1 & $0,281 \pm 0,312$ \\
Nybelinia sp. & 0 & 0 & $0,045 \pm 0,082$ \\
Contracaecum sp. & 2 & 0 & $0,042 \pm 0,135$ \\
Pseudoterranova sp. & 2 & 0 & $0,051 \pm 0,160$ \\
\hline
\end{tabular}


Tabela V. Índice de dispersão (ID) e indice de agregação de Green (IG) dos metazoários parasitos de Caranx hippos, do litoral do estado do Rio de Janeiro, Brasil.

\begin{tabular}{lcc}
\hline \multicolumn{1}{c}{ Parasitos } & Indice de dispersão & Indice de agregação de Green \\
\hline Allopyragraphorus hippos & 6,31 & 0,09 \\
Cemocotyllela elongata & 4,58 & 0,06 \\
Cemocotyle noveboracensis & 16,17 & 0,25 \\
Protomicrocotyle mirabilis & 3,18 & 0,03 \\
Bucephalus varicus & 42,76 & 0,71 \\
Manteria brachyderus & 19,33 & 0,31 \\
Parahemiurus merus & 16,75 & 0,27 \\
Cucullanus pulcherrimus & 3,27 & 0,03 \\
Caligus robustus & 1,95 & 0,01 \\
Lernanthropus giganteus & 2,93 & 0,03 \\
\hline
\end{tabular}

Tabela VI. Índice de dispersão (ID) e indice de agregação de Green (IG) dos metazoários parasitos de Caranx latus, do litoral do estado do Rio de Janeiro, Brasil.

\begin{tabular}{lcc}
\hline \multicolumn{1}{c}{ Parasitos } & Indice de dispersão & Indice de agregação de Green \\
\hline Bucephalus varicus & 14,67 & 0,25 \\
Parahemiurus merus & 6,35 & 0,09 \\
Tergestia pectinata & 9,17 & 0,15 \\
Cemocotyle carangis & 14,98 & 0,25 \\
Pseudomazocraes selene & 3,32 & 0,04 \\
Nybelinia sp. & 7,19 & 0,11 \\
Contracaecum sp. & 10,35 & 0,17 \\
Pseudoterranova sp. & 49,79 & 0,91 \\
\hline
\end{tabular}

Em C. hippos, o comprimento total dos espécimes machos $(\mathrm{N}=35)$ e das fêmeas $(\mathrm{N}=25)$, não apresentaram diferença significativa $(t=-0,677, P=0,501)$. Em $C$. latus também não houve diferença significativa no comprimento total de machos $(\mathrm{N}=25)$ e fêmeas $(\mathrm{N}=30)(t=0,351, P=0,727)$. Dentre os metazoários parasitos de $C$. hippos cinco espécies apresentaram correlação positiva entre o comprimento total do hospedeiro e a abundância parasitária. E apenas o digenético Bucephalus varicus Manter, 1940 apresentou correlação positiva entre a prevalência parasitária e o comprimento total do hospedeiro (Tab. VII).

Tabela VII. Valores do coeficiente de correlação por postos de Spearman ( $r s$ ) e do coeficiente de correlação de Pearson $(r)$ para avaliar o relacionamento entre o comprimento total de Caranx hippos e a abundância e prevalência dos componentes de sua comunidade parasitária ( $P=$ nível de significância).

\begin{tabular}{|c|c|c|c|c|}
\hline Parasitos & rs & $P$ & $r$ & $P$ \\
\hline Bucephalus varicus & $0,268 *$ & 0,038 & $0,965 *$ & 0,039 \\
\hline Manteria brachyderus & 0,062 & 0,639 & 0,548 & 0,415 \\
\hline Parahemiurus merus & 0,192 & 0,141 & 0,180 & 0,820 \\
\hline Allopyragraphorus hippos & $0,296 *$ & 0,021 & 0,401 & 0,599 \\
\hline Cemocotyllela elongata & 0,204 & 0,117 & 0,686 & 0,314 \\
\hline Cemocotyle noveboracensis & $-0,202$ & 0,121 & $-0,767$ & 0,233 \\
\hline Protomicrocotyle mirabilis & $0,275^{*}$ & 0,033 & 0,599 & 0,401 \\
\hline Cucullanus pulcherrimus & $0,423^{*}$ & $<0,001$ & 0,765 & 0,235 \\
\hline Caligus robustus & 0,096 & 0,463 & 0,388 & 0,612 \\
\hline Lernanthropus giganteus & $0,508^{*}$ & $<0,001$ & 0,795 & 0,205 \\
\hline
\end{tabular}

(*) Valores significativos.

Revta bras. Zool. 18 (2): 399 - 410, 2001 
Quatro espécies de metazoários parasitos de C. latus apresentaram relação entre a abundância parasitária e o comprimento total do hospedeiro: B. varicus e Tergestia pectinata (Linton, 1905) correlação negativa, e C. carangis e Pseudoterranova sp. correlação positiva. Duas espécies apresentaram relação entre a prevalência parasitária e o comprimento total do hospedeiro: $T$. pectinata correlação negativa e Pseudoterranova sp. correlação positiva (Tab. VIII).

Tabela VIII. Valores do coeficiente de correlação por postos de Spearman ( $r$ s) e do coeficiente de correlação de Pearson ( $r$ ) para avaliar o relacionamento entre o comprimento total de Caranx latus e a abundância e prevalência dos componentes de sua comunidade parasitária ( $P=$ nivel de significância).

\begin{tabular}{lcccc}
\hline \multicolumn{1}{c}{ Parasitos } & \multicolumn{1}{c}{$r$ s } & $P$ & $r$ & $P$ \\
\hline Bucephalus varicus & $-0,350^{*}$ & 0,009 & $-0,804$ & 0,101 \\
Parahemiurus merus & $-0,010^{*}$ & 0,943 & 0,383 & 0,589 \\
Tergestia pectinata & $-0,426^{*}$ & 0,001 & $-0,891^{*}$ & 0,043 \\
Cemocotyle carangis & $0,564^{*}$ & $<0,001$ & 0,573 & 0,313 \\
Pseudomazocraes selene & $0,027^{*}$ & 0,844 & 0,080 & 0,899 \\
Nybelinia sp. & 0,224 & 0,100 & 0,624 & 0,261 \\
Contracaecum sp. & 0,118 & 0,392 & 0,586 & 0,299 \\
Pseudoterranova sp. & $0,368^{*}$ & 0,005 & $0,895^{*}$ & 0,040 \\
\hline
\end{tabular}

$\left(^{\star}\right)$ Valores significativos.

Apenas o copépode Caligus robustus Bassett-Smith, 1898, parasito de $C$. hippos, apresentou diferenças para os valores de abundância (machos: 0,1; fềmeas: 0,5 ) e prevalência parasitária (machos: $5,7 \%$; fềmeas: $32 \%$ ) com relação ao sexo dos hospedeiros $(Z=-2,554, P=0,011 ; F=0,012)$. Nenhum componente da fauna parasitária de $C$. latus apresentou correlação entre o sexo e a abundância e prevalência parasitária.

\section{Infracomunidades parasitárias}

Todos os espécimes de C. hippos examinados estavam parasitados com pelo menos, uma espécie de metazoário. Foram coletados um total de 1939 parasitos com média de 32,3 $\pm 85,5$ por peixe. Dos 55 espécimes de C. latus examinados, 53 $(96,4 \%)$, estavam parasitados com pelo menos uma espécie de metazoário, sendo coletados um total de 1486 parasitos com média de $27 \pm 25,2$ por peixe. A diferença entre o número de parasitos por peixe do xaréu e do xerelete foi estatísticamente significativa $(Z=-2,272, P=0,023)$.

O número total de parasitos por peixe apresentou correlação positiva com o comprimento total de $C$. hippos $(r s=0,564 ; P<0,001)$ e de C. latus $(r s=0,339 ; P$ $=0,011)$ e não mostrou qualquer correlação com o sexo de ambos hospedeiros $(Z$ $=-1,562, P=0,118 ; Z=-0,617, P=0,537)$, respectivamente. A distribuição do número total de parasitos por peixe foi altamente dispersa para C. hippos (ID = 226,45 ) e para $C$. latus (ID $=23,49$ ).

As infracomunidades parasitárias de C. hippos tiveram uma diversidade média de $H=0,555 \pm 0,407$ e uma diversidade máxima de 1,363. O índice de uniformidade de Brillouin $(J)$ teve uma média de 0,521 $\pm 0,319$. A diversidade parasitária mostrou-se fortemente correlacionada com o comprimento total do 
hospedeiro ( $r s=0,735, P<0,001)$ e não apresentou correlação com o sexo do hospedeiro $(Z=-0,460 ; P=0,646)$. As infracomunidades parasitárias de $C$. latus tiveram uma diversidade média de $H=0,608 \pm 0,356$ e uma diversidade máxima de 1,258 . O índice de uniformidade de Brillouin $(J)$ teve uma média de $0,577 \pm 0,275$. A diversidade parasitária não mostrou correlação com o comprimento total do hospedeiro $(r s=0,216 ; P<0,113)$ e também não apresentou correlação com o sexo do hospedeiro $(Z=-1,438 ; P=0,150)$.

A riqueza parasitária do xaréu apresentou uma média de $3,1 \pm 1,8$, com amplitude de variação de 1-10. Doze hospedeiros (20\%) apresentaram infecção por uma espécie de metazoário, 12 (20\%), 17 (28,3\%), $8(13,3 \%), 4(6,7 \%), 5(8,3 \%)$, $1(1,7 \%)$ e $1(1,7 \%)$ tiveram infecções múltiplas por 2,3,4,5,6,7 e 10 espécies, respectivamente (Fig. 1). A riqueza parasitária do xerelete apresentou uma média de $2,9 \pm 1,6$, com amplitude de variação de 1-8. Cinco hospedeiros $(9,1 \%)$ apresentaram infecção por uma espécie de metazoário, 19 (34,5\%), 9 (16,4\%), $12(21,8 \%)$, $5(9,1 \%), 1(1,8 \%), 1(1,8 \%)$ e $1(1,8 \%)$ tiveram infecções múltiplas por 2,3,4,5,6,7 e 8 espécies, respectivamente (Fig. 2). Não houveram diferenças significativas entre a riqueza parasitária $(Z=-0,183, P=0,855)$ e a diversidade parasitária $(Z=-0,755$, $P=0,450)$ de $C$. hippos e $C$. latus.

As infrapopulações parasitárias foram separadas em dois grupos: ectoparasitos (monogenéticos e copépodes) e endoparasitos (digenéticos, cestóides e nematóides). Apenas um par de ectoparasitos A. hippos-Protomicrocotyle mirabilis (MacCallum, 1918) apresentou co-ocorrência e covariação positiva $(r s=0,386, P$ $\left.=0,002 ; X^{2}=12,09, P<0,001\right)$ em $C$. hippos. Em $C$. latus duas espécies de endoparasitos $B$. varicus - Parahemiurus merus (Linton, 1910) ( $r s=-0,375, P=$ 0,$\left.005 ; X^{2}=4,04, P=0,044\right)$ apresentaram co-ocorrência negativa e covariação positiva; $B$. varicus - Pseudoterranova $\mathrm{sp}$. $\left(r s=0,319, P=0,017 ; X^{2}=6,50, P=\right.$ $0,011)$ apresentaram co-ocorrência e covariação positiva.

As comunidades parasitárias de C. hippos e C. latus apresentaram um valor de 55,5 para o coeficiente de similaridade de Sorenson e de 30,4 para o coeficiente de similaridade percentual.

\section{DISCUSSÃO}

Os resultados obtidos no presente trabalho indicam a presença de alguns padrões na composição das infracomunidades parasitárias do xaréu e do xerelete: 1) dominância dos endoparasitos; 2) relacionamento da abundância e prevalência dos parasitos com o tamanho dos hospedeiros; 3) ausência, a nível das infracomunidades, de correlação da prevalência e abundância parasitária com o sexo dos hospedeiros; 4) escassez de relacionamentos parasitários interespecíficos; 5) presença de larvas de anisakídeos com baixa prevalência e abundância parasitária, localizadas apenas no mesentério dos hospedeiros.

A dominância dos endoparasitos é um padrão já detectado nas infracomunidades parasitárias de outros peixes pelágicos da região sudeste do litoral brasileiro. TAKEMOTO et al. (1996) demonstraram que os trematódeos digenéticos são o táxon predominante nas comunidades parasitárias das guaiviras (Oligoplites spp.) e LUQUE \& CHAVES (1999) mencionaram também este padrão nas infracomunidades

Revta bras. Zool. 18 (2): 399 - 410, 2001 


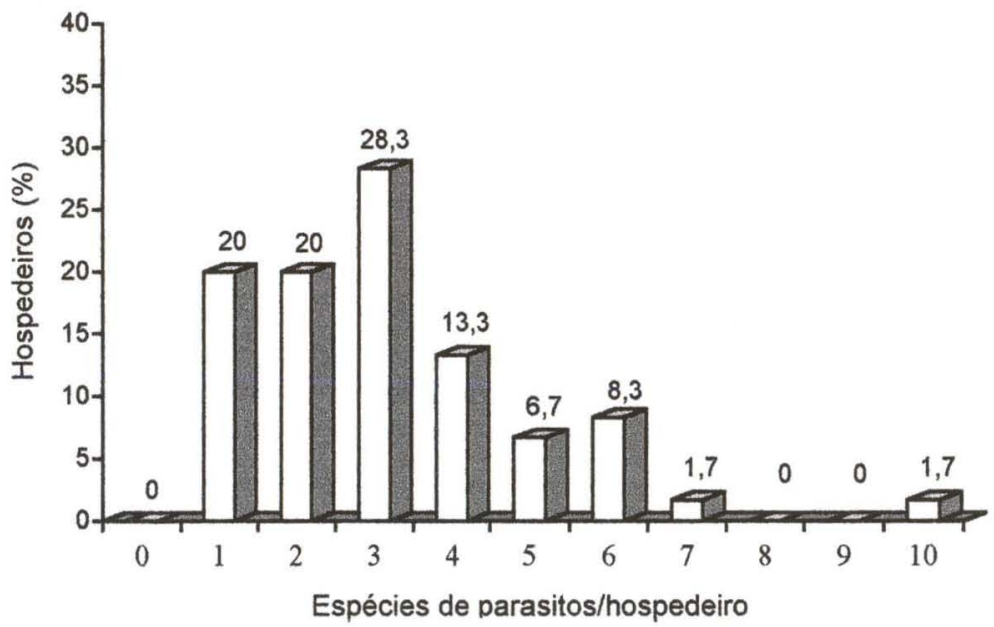

Fig. 1. Distribuição do número de componentes das infracomunidades de metazoários parasitos de Caranx hippos no litoral do estado do Rio de Janeiro, Brasil.

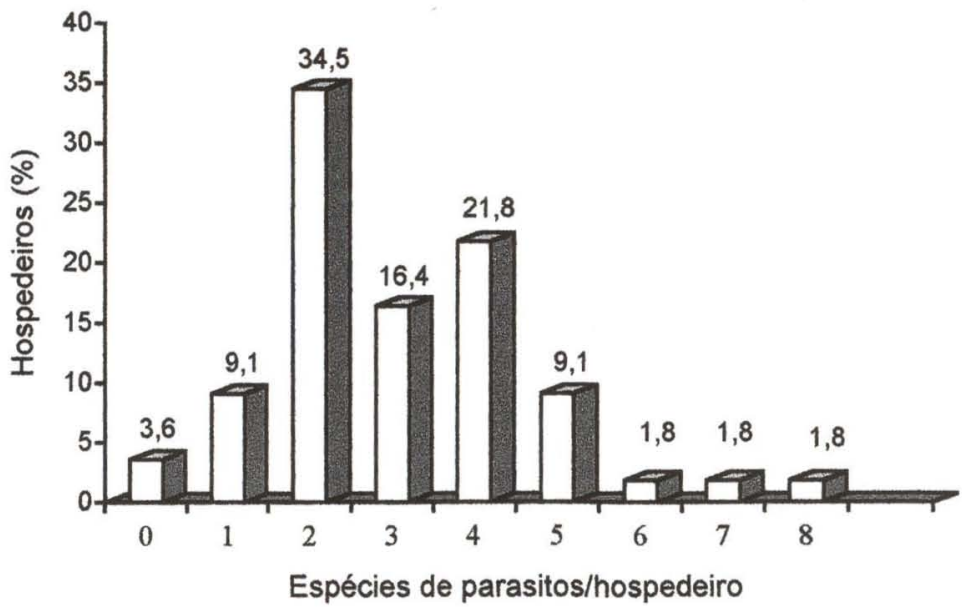

Fig. 2. Distribuição do número de componentes das infracomunidades de metazoários parasitos de Caranx latus no litoral do estado do Rio de Janeiro, Brasil.

parasitárias das anchovas (Pomatomus saltator). No xaréu e no xerelete, a presença de endoparasitos transmitidos troficamente, pode ser favorecida pelos hábitos alimentares destas espécies. De acordo com MENEZES \& FigueIrEdo (1980), o xaréu e o xerelete têm hábitos carnívoros, sendo ictiófagos e se alimentando em menor escala de crustáceos e invertebrados planctônicos. O hábito de formar cardumes perto da costa para encurralar sua presas revela também suas características gregárias que podem facilitar a transmissão de ectoparasitos com ciclo de vida holoxeno como os monogenéticos e os copépodes (CARVALHO-FilHo 1999). En- 
tretanto, segundo LUQUE \& CHAVES (1999), na maioria dos peixes estudados no litoral do estado do Rio de Janeiro, foi encontrada sempre dominância dos endoparasitos independentemente do caráter bentônico ou pelágico dos hospedeiros.

A influência do tamanho do hospedeiro sobre a composição qualitativa e quantitativa das infracomunidades parasitárias, é um tópico bastante documentado. SAAD-FAARES \& COMBES (1992), LUQue et al. (1996) e LuQUE \& CHAVES (1999), chamaram a atenção para que se evite generalizações à respeito. $\mathrm{O}$ parasitismo não necessariamente, aumenta nos peixes maiores devido a um processo mecânico de acumulação e de maior tempo de exposição às infecções. Assim como nos carangídeos do gênero Oligoplites, a heterogeneidade de padrões na relação tamanho do hospedeiro/prevalência e abundância parasitária encontrada no xaréu e no xerelete também é notória, e sua análise resulta insuficiente devido à falta de trabalhos relacionados com a biologia e aspectos populacionais de C. hippos e C. latus no litoral sudeste brasileiro.

A literatura sobre peixes marinhos, indica que muitos parasitos não apresentam variações quantitativas em relação ao sexo do hospedeiro, e isto é considerado um reflexo da ausência de diferenças na biologia e na dinâmica populacional entre os hospedeiros machos e fêmeas (LUQUE et al. 1996). Porém, é necessário um enfoque experimental para detectar a importância de alguns aspectos fisiológicos (hormonais e imunológicos), morfológicos e comportamentais dos hospedeiros (BUNDY 1988; LADLE 1992, POULIN 1996). No presente trabalho repete-se o mesmo padrão encontrado por TAKEMOTO et al. (1996) para Oligoplites spp., em cujas comunidades parasitárias não foi detectada influência do sexo dos hospedeiros sobre a maioria dos componentes.

É importante mencionar que existe similaridade entre as espécies de guaiviras (Oligoplites spp.) examinadas por TAKEMOTO et al. (1996) e os peixes do gênero Caranx analisados no presente trabalho, principalmente em relação às infracomunidades de endoparasitos. Todas as espécies de trematódeos digenéticos registradas por TAKEMOTO et al. (1996) foram encontradas também em C. hippos e C. latus. Foi confirmada também a similaridade esperada entre as comunidades parasitárias do xaréu e do xerelete, principalmente em relação as espécies de parasitos transmitidos troficamente. Isto poderia ser explicado por possíveis similaridades nos hábitos alimentares destes carangídeos, tal como sugerido por LUQUE et al. (1996) que registraram uma alta similaridade na composição das comunidades parasitárias de duas espécies de haemulídeos do Rio de Janeiro.

Também, é notória a diferença existente entre a composição das infracomunidades de ectoparasitos, principalmente monogenéticos, nos carangídeos do estado do Rio de Janeiro (Oligoplites spp., Caranx spp. e Selene vomer (Linnaeus, 1758)) (TAKEMOTO et al. 1996; CEZAR et al. 2000; LUQUE et al. 2000), onde não foram encontradas espécies parasitando mais de um hospedeiro, com a exceção de $P$ seudomazocraes selene Hargis, 1957 ectoparasito de Selene vomer (Linnaeus, 1758) encontrado também parasitando C. latus. Isto confirma a alta especificidade pelo hospedeiro mostrada pelos monogenéticos parasitos de carangídeos.

BASHIRULLAH \& RODRIGUEZ (1992) quantificaram a presença de quatro espécies de monogenéticos em C. hippos da Venezuela, encontrando vários pares de espécies associadas significativamente. No presente trabalho também foram 
coletadas estas quatro espécies de monogenéticos, entretanto, com prevalência e abundância menores e apenas com um par de espécies associadas. A falta de trabalhos sobre a dinâmica populacional do xaréu no Brasil e na Venezuela dificulta a avaliação e interpretação deste tipo de resultado parasitológico.

Um outro aspecto a mencionar, devido às suas repercussões para estudos biogeográficos, é a maior riqueza e diversidade parasitária encontradas em carangídeos do Oceano Atlântico da América do Sul em relação às espécies de carangídeos estudadas no Oceano Pacífico do mesmo continente (OliVA 1994). Diferenças anfioceánicas já tinham sido registradas para sciaenídeos por LUQUE \& OLIVA (1999) que sugeriram a influência de perturbações ecológicas, como o fenômeno "El Niño" e ressurgências oceânicas, sobre a estrutura das comunidades parasitárias de peixes marinhos da América do Sul.

A presença de larvas de nematóides anisakídeos com baixa prevalência e abundância parasitária e localizadas apenas no mesentério dos peixes estudados, indica que o potencial zoonótico das mesmas é muito baixo, não existindo perigo de infecção em humanos, devido à ausência das larvas de anisakídeos na musculatura somática do xaréu e do xerelete.

Como foi observado nas espécies de parasitos de Oligoplites spp. estudadas por TAKEMOTO et al. (1996), a escassez de relacionamentos interespecíficos encontrados entre os componentes das infracomunidades parasitárias de C. hippos e de C. latus, reforçam o postulado de que as comunidades parasitárias de peixes marinhos são na realidade complexos insaturados e pouco ordenados de espécies, fugindo dos padrões interativos comprovados para outros grupos de hospedeiros, principalmente aves de ambientes aquáticos (ROHDE et al. 1995) e mencionado para peixes marinhos do litoral brasileiro por CEZAR \& LUQUE (1999).

Estudos adicionais sobre as comunidades parasitárias das outras espécies de carangídeos do Oceano Atlântico e Pacífico da América do Sul são necessários para que se possa avaliar e confirmar padrões na estrutura das comunidades parasitárias destes peixes na Região Neotropical.

\section{REFERÊNCIAS BIBLIOGRÁFICAS}

Amato, J.F.R. 1982. Digenetic Trematodes of Percoid fishes of Florianópolis, southern Brasil Fellodistomidae, Monascidae, Diplangidae, Zoogonidae, and Waretrematidae with description of two new species. Rev. Brasil. Biol. 42: 681-699.

1983. Digenetic Trematodes of Percoid fishes of Florianópolis, southern Brasil Pleorchiidae, Didymozoidae and Hemiuridae, with the description of three new species. Rev. Brasil. Biol. 43: 99-124.

Bashirullah, A.K.M. \& J.C. Rodriguez. 1992. Spatial distribution and interrelationships of four Monogenoidea of Jack mackerel Caranx hippos (Carangidae) in the north-east of Venezuela. Acta

Cient. Venezol. 43: 125-128.

Bundy, D.A.P. 1988. Sexual effects on parasite infection. Parasitol. Today 4: 186-189.

BUSH, A.O.; J.M. AHO \& C.R. KENNEDY. 1990. Ecological versus phylogenetic determinants of helminth parasite community richness. Evol. Ecol. 4: 1-20.

Bush, J.O.; K.D. LAFFerTy; J.M. Lotz \& A.W. Shostak. 1997. Parasitology meets ecology on its own terms: Margolis et al. revisited. Jour. Parasitol. 83: 575-583.

Carvalho-Filho, A. 1999. Peixes da costa brasileira. São Paulo, Ed. Marca D’água, 320p.

Cezar, A.D. \& J.L. Luque. 1999. Metazoan parasites of the Atlantic Spadefish, Chaetodipterus faber

Revta bras. Zool. 18 (2): 399 - 410, 2001 
(Teleostei: Ephippidae) from the coastal zone of the State of Rio de Janeiro, Brazil. Jour. Helminthol. Soc. Wash. 66: 14-20.

Cezar, A.D.; J.L. Luque \& N.D. Chaves. 2000. Aspectos quantitativos das infracomunidades de metazoários ectoparasitos do peixe-galo Selene vomer (Osteichthyes: Carangidae) do litoral do Estado do Rio de Janeiro. Contrib. Avulsas Hist. Nat. Brasil, Sér. Zool., 16: 1-7.

FERNANDES, B.M.M.; A. KOHN \& R.M. PINTO. 1985. Aspidogastrid and digenetic trematodes parasites of marine fishes of the coast of Rio de Janeiro State, Brazil. Rev. Brasil. Biol. 45: 109-116.

Holmes, J.C. \& R. Podesta. 1968. The helminths of wolves and coyotes from the forested regions of Alberta. Can. Jour. Zool. 46: 1193-1204.

Kohn, A.; C.P. SAntos \& M.F.D. BAPTISTA-FARIAS. 1992. New host records and localities of some monogenea from Brazilian marine fishes with scanning electron microscopy of Bicotylophora trachinoti (Mac Callum, 1921). Mem. Inst. Oswaldo Cruz 87 (Suppl. 1): 109-114.

LADLE, R.J. 1992. Parasites and sex: catching the Red Queen. Trends Ecol. Evol. 7: 405-408.

LUDWIG, J.A. \& J.F. REYNOLDS. 1988. Statistical Ecology: A primer on methods and computing. New York, Wiley-Interscience Publications, 337p.

LuQue, J.L. \& N.D. Chaves. 1999. Ecologia da comunidade de metazoários parasitos de Pomatomus saltator (Osteichthyes: Pomatomidae) do litoral do Estado do Rio de Janeiro. Rev. Brasil. Zool. 16: 711-723.

Luque, J.L. \& M.E. Oliva. 1999. Metazoan parasite infracommunities of Menticirrhus (Teleostei: Sciaenidae): an amphi-oceanic approximation. Jour. Parasitol. 85: 379-381.

LuQue, J.L.; D.R. Alves \& C.S. Sabas. 2000. Metazoários parasitos do xaréu Caranx hippos e do xerelete Caranx latus (Osteichthyes: Carangidae) do litoral do Estado do Rio de Janeiro. Contrib. Avulsas Hist. Nat. Brasil, Sér. Zool., 25: 1-17.

Luque, J.L.; J.F.R. Amato \& R.M. TAKEмоTo. 1996. Comparative analysis of the communities of metazoan parasites of Orthopristis ruber and Haemulon steindachneri (Osteichthyes: Haemulidae) from the southeastern Brazilian littoral: I. structure and influence of the size and sex of hosts. Rev. Brasil. Biol. 56: 279-292.

Menezes, N.A. \& J.L. Figueiredo. 1980. Manual de Peixes Marinhos do Sudeste de Brasil IV. Teleostei (3). São Paulo, Museu de Zoologia, Univ. de São Paulo, 96p.

NerAasen, T.G. \& J.C. Holmes. 1975. The circulation of cestodes among three species of geese nesting on the Anderson River Delta. Acta Parasitol. Polon. 23: 277-289.

Oliva, M.E. 1994. Parasites of the Chilean Jack Mackerel Trachurus symmetricus murphyi (Pisces: Carangidae). Mem. Inst. Oswaldo Cruz 89: 363-364.

Poulin, R. 1996. Sexual inequalities in helminth infections: a cost of being a male. Amer. Nat. 147: 287-295.

ROHDE, K. 1991. Intra- and interspecific interactions in low density populations in resource-rich habitats. Oikos 60: 91-104.

RoHDE, K.; C. HAYWARD \& M. HEAP. 1995. Aspects of the ecology of metazoan ectoparasites of marine fishes. Int. Jour. Parasitol. 25: 945-970.

SAAD-FARES, A. \& C. COMBES. 1992. Abundance/host size relationships in a fish trematode community. Jour. Helminthol. 66: 187-192.

TAKEMOTO, R.M., J.F.R. AMATO \& J.L. LUQUE. 1996. Comparative analysis of the metazoan parasite communities of leatherjackets, Oligoplites palometa, $O$. saurus and $O$. saliens (Osteichthyes: Carangidae) from Sepetiba Bay, Rio de Janeiro, Brazil. Rev. Brasil. Biol. 56: 639-650.

Travassos, L.P.; J.F.T. Freitas \& P.F. Bührnheim. 1966. Trematódeos de peixes do litoral capixaba: "Separogermiductus zeloticus" sp. n., parasita de xaréu. Atas Soc. Biol. Rio de Janeiro 10: 35-38.

ZAR, J.H. 1996. Biostatistical Analysis. New Jersey, Prentice-Hall, Inc., Upper Saddle River, $3^{\text {rd }}$ ed., $662 \mathrm{p}$.

Recebido em 23.III.2000; aceito em 15.V.2001. 